\title{
The Electric Work Method. III. Electrokinetic Effects
}

\author{
Herman Gerhard Hertz and Signe Kjelstrup Ratkje* \\ Institute of Physical Chemistry and Electrochemistry, University of Karlsruhe, Kaiserstrasse 12, D-7500 Karlsruhe, Germany
}

\begin{abstract}
Hertz, H. G. and Ratkje, S. K., 1991. The Electric Work Method. III. Electrokinetic Effects. - Acta Chem. Scand. 45: 129-137.

A new theory of electrokinetic effects has been derived. The theory represents an extension of the electric work method described earlier [cf. Hertz, H. G. and Ratkje, S. K. Acta Chem. Scand. 44 (1990) 542 and 554; J. Electrochem. Soc. 136 (1989) 1692 and 1698]. The extension is obtained by including the conservation equation for momentum into the analysis. The theory includes all basic formulas for electrokinetic effects as derived in the literature so far. The theory does not, however, use Onsager reciprocal relations or other assumptions to derive relations between these formulas. In addition, a new equation is obtained: a description of the phenomenon "friction electricity". Thus we claim that our theory is more general than others. Friction electricity appears as a result of excess momentum transference due to the relative movement of different materials of the system. Momentum transference factors are introduced to describe the phenomenon. These parameters replace the zeta potential, $\zeta$, or the water transference number in the description of the EMF. Such an alternative is needed for leaky membranes.
\end{abstract}

The purpose of the present paper is to derive equations for energy conversion in a system where viscous flows are connected with electric current. We thus extend the range of a new electrochemical method which has recently been published $^{1-4}$ and which did not yet include the momentum conservation law. The method has been named the electric work method. ${ }^{4}$

The electric work method deals with systems on a strictly phenomenological basis. All fundamental formulas for electric potentials (EMFS) are derived from this basis. Conventional EMFS are derived from electrostatic considerations (see e.g. Bockris and Reddy). ${ }^{5}$ Electrokinetic effects are also extensively treated in the field of thermodynamics of irreversible processes. ${ }^{6-9}$ We shall use the conservation laws for mass, energy and momentum, together with specific phenomenological equations to describe the macroscopic motion of mass in electrochemical systems. The conservation law for momentum, the equation of motion, will be used to replace partly the pressure gradient as a driving force into the theory. A discussion of the momentum conservation law in the absence and presence of electric current provides a basis for the analysis.

From this basis we shall show that (1) Onsager receprocal relations are not needed to connect, e.g. the electroosmotic flux and the streaming potential, and (2) the zeta potential has an alternative interpretation as the underlying cause for the EMF. More important, however, is the deriv-

\footnotetext{
* To whom correspondence should be addressed. Permanent address: Division of Physical Chemistry, Norwegian Institute of Technology, University of Trondheim, N-7034 Trondheim-NTH, Norway.
}

ation of a new equation which describes a phenomenon which we call "friction electricity". The equation relates the electric work (EMF) to defined momentum transference factors at the electrodes and at the walls. Momentum transference factors are derived from the momentum conservation law. From the equation for friction electricity all other basic formulas can be derived.

The need for a new electrochemistry has been discussed in general terms in our introductory paper. ${ }^{1}$ The conventional treatment of electrokinetic phenomena ${ }^{5}$ possesses the same general difficulties as discussed there, as well as some specific ones. For instance, experimental results for streaming potentials are commonly interpreted as an electrostatic potential perpendicular to the direction of the electric current, the zeta potential. The zeta potential varies across the pore to produce an EMF in the transport direction. This potential is in principle an unmeasurable quantity, so its existence cannot be verified. No operational distinction between the zeta potential and other surface potentials exists. Assumptions regarding the zeta potential are not then amenable to experimental control.

Instead of relating the EMF of electrochemical systems to electrostatic potentials in the cell, we have started to interpret the EMF, or the electric work performed in the external circuit, by the mass and accompanying energy changes in the system..$^{1-4} \mathrm{~A}$ similar approach was taken by Førland et al., ${ }^{9}$ however, within the context of irreversible thermodynamics and by assuming that Onsager reciprocal relations are valid. Both approaches imply that each part of the system is described by measurable quantities only. Here we pursue the electric work method ${ }^{1-4}$ by studying also the effect of momentum changes. 


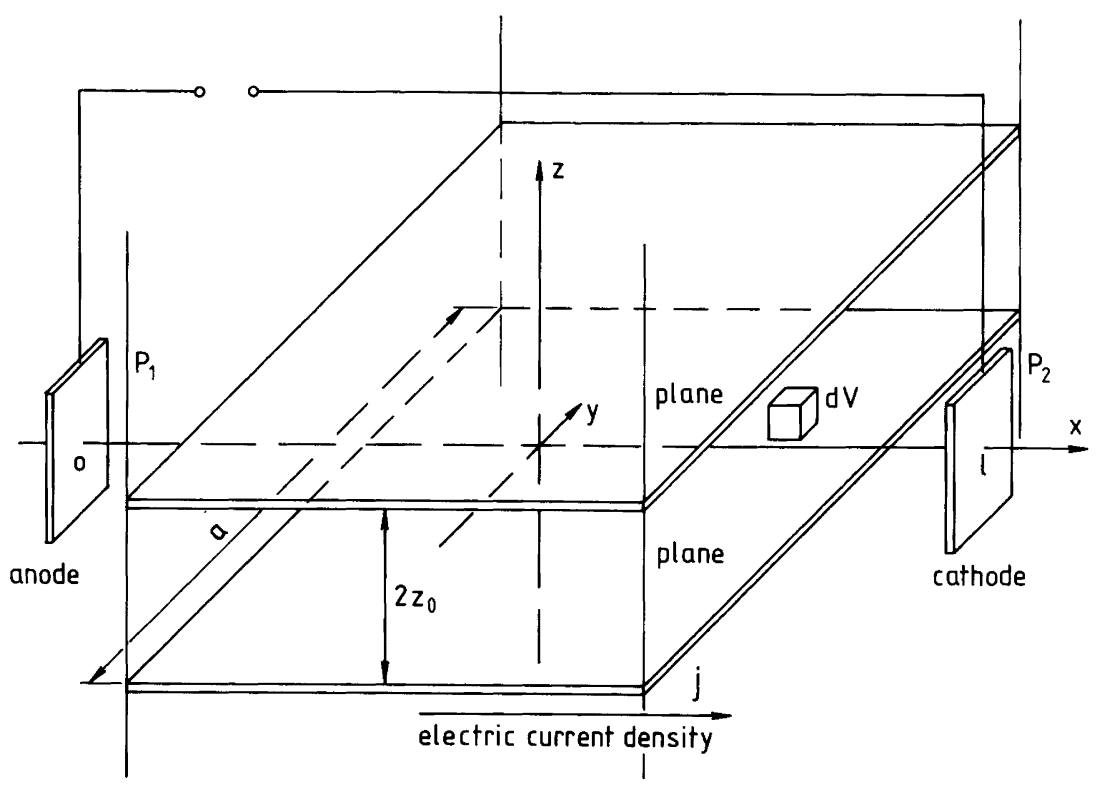

Fig. 1. Schematic illustration of the system.

\section{Momentum conservation in the absence of electric current}

To bring out the essential features of the problem we choose a model system which is as simple as possible. Consider a planar slit separating two salt solutions, one on the left-hand side, the other on the right-hand side of the slit (Fig. 1). The only pathway for the liquid from one solution to the other is between planes in the $x$-direction. Consider a flowing liquid within this slit. We shall consider the $x y$-planes as having a large extension, $a$, in the $y$-direction. The extension in the $x$-direction is 1 . The distance between the planes is $2 z_{0}$, with $2 z_{0}<<a$.

The distance between the planes is such that it allows for bulk transport of liquid. A pressure difference may exist between the solutions. Two electrodes, reversible to $\mathrm{Cl}$, are used to pass electric current through the system. The electrodes have finite dimensions in all directions, as indicated in the figure. The concentration of the salt, $\mathrm{NaCl}$, is the same in the two solutions. We shall assume that the effect of gravity is insignificant.

The components of a surface element vector within this system, $\mathrm{d} \boldsymbol{A}$, can be written $\mathrm{d} \boldsymbol{A}=\left\{\mathrm{d} \boldsymbol{A}_{x}, \mathrm{~d} \boldsymbol{A}_{y}, \mathrm{~d} \boldsymbol{A}_{z}\right\}$. An illustration is given in Fig. 2 . The balance equation for momentum transport is the same as the equation of motion from hydrodynamic theory, see e.g. Kreuzer. ${ }^{10}$ For an incompressible liquid moving with a velocity $v$ relative to the wall we have eqn. (1), where $\varrho$ is the mass density and $t$ is

$\mathrm{Q}(\mathrm{d} v / \mathrm{d} t)=-\operatorname{Div} \cap$

the time. The left-hand side of the equation is the change in momentum per unit volume and unit time in the volume element $\mathrm{d} V$. A volume element is shown in Fig. 1. The Div term contains the stress tensor $n$. The properties of $\cap$ are explained in basic hydrodynamic theory, see e.g. Ref. 10; here we only give some facts needed below. The component representation of $n$ may be written as in eqn. (2). The

$$
\cap=\left[\begin{array}{lll}
n_{x x} & n_{x y} & n_{x z} \\
n_{y x} & n_{y y} & n_{y z} \\
n_{z x} & n_{z y} & n_{z z}
\end{array}\right]
$$

tensor matrix is symmetric in a liquid without rotation, as here. The tensor connects the transported quantity "momentum" per unit time, i.e. the force $\boldsymbol{F}$, to $\mathrm{d} \boldsymbol{A}$, so that $\mathrm{d} \boldsymbol{F}=$ $n \mathrm{~d} A$ or as in eqn. (3), where $\mathrm{d} F_{x}$ is the $x$-component of the

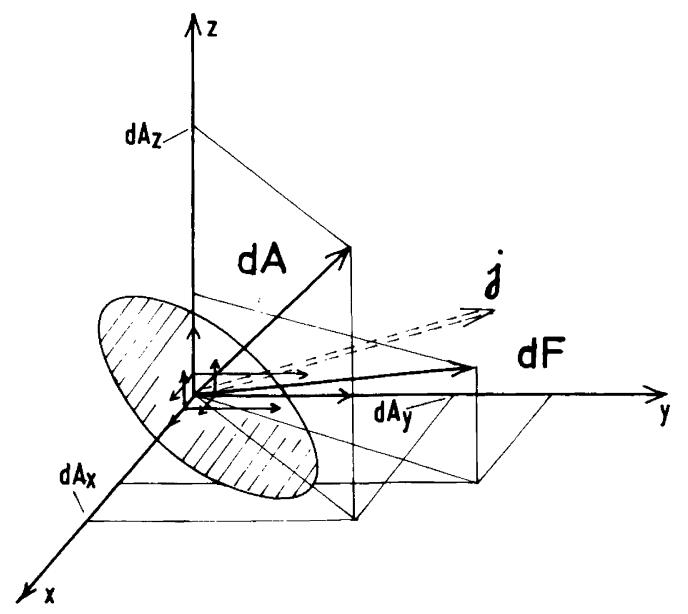

Fig. 2. Surface area vector $\mathrm{d} \mathbf{A}$ with components, and the current density vector, $\boldsymbol{j}$. From $\boldsymbol{j}$ a tensor is constructed which assigns the force vector $\mathrm{d} \boldsymbol{F}$ to $\mathrm{d} \boldsymbol{A}$. The three coordinate triples at the origin refer to the forces through $\mathrm{d} A_{x}, \mathrm{~d} A_{y}$ and $\mathrm{d} A_{z}$, respectively. 
$\mathrm{d} F_{x}=\cap_{x x} \mathrm{~d} A_{x}+\cap_{x y} \mathrm{~d} A_{y}+\cap_{x z} \mathrm{~d} A_{z}$

$\mathrm{d} F_{y}=\cap_{y x} \mathrm{~d} A_{x}+\cap_{y y} \mathrm{~d} A_{y}+\cap_{y z} \mathrm{~d} A_{z}$

$\mathrm{d} F_{z}=\cap_{z x} \mathrm{~d} A_{x}+\cap_{z y} \mathrm{~d} A_{y}+\cap_{z z} \mathrm{~d} A_{z}$

total momentum per unit time going through the area $\mathrm{d} \boldsymbol{A}$. If $\mathrm{d} \boldsymbol{A}=\{1,0,0\}$, then $\boldsymbol{F}=\left\{\cap_{x x}, \cap_{y x}, \cap_{z x}\right\}$. The shear stress components, $\cap_{i j}$, express the transfer of momentum due to statistical molecular movement between layers in the liquid. The off-diagonal elements represent transfer of momentum from direction $i$ through a surface element of direction $j$. The diagonal components of the tensor are the normal stresses (the hydrostatic pressure, $p$, and the viscous resistance to compression). The viscous resistance to compression is zero for incompressible liquids. Thus we have eqn. (4), where $\{\mathbf{1}\}$ is the unit tensor and eqn. (5)

$\mathrm{n}=p\{\mathbf{1}\}+\mathrm{n}^{\prime}$

$p=\left(\cap_{x x}+\cap_{y y}+\cap_{z z}\right) / 3$

$\bigcap_{x x}=\cap_{y y}=n_{z z}=p$

holds. We limit outselves to Newtonian liquids with viscosity $\eta$. The $y x$ - and $z x$-components of the shear stress are then given by eqn. (6). ${ }^{10}$ We write these equations to show

$n_{y x}=-2 \eta\left(\partial v_{x} / \partial y+\partial v_{y} / \partial x\right) / 2$

$n_{z x}=-2 \eta\left(\partial v_{x} / \partial z+\partial v_{z} / \partial x\right) / 2$

the similarity with tensor components which will appear below.

The local balance of momentum for viscous flow in the absence of electric current in the $x$-direction is given by eqn. (7), derived from eqns. (1)-(5) with $\partial^{2} v_{x} / \partial x \partial z=$ $\partial^{2} v_{x \partial} z \partial x=0$.

$\mathrm{\rho}\left(\mathrm{d} v_{x} / \mathrm{d} t\right)=-(\operatorname{Div} \cap)_{x}=-\mathrm{d} p / \mathrm{d} x+\eta\left(\partial^{2} v_{x} / \partial z^{2}\right)$

The divergence has been formed according to eqn. (8), and

$\operatorname{Div} \cap=\{\partial / \partial x, \partial / \partial y, \partial / \partial z\}\left[\begin{array}{lll}\cap_{x x} & n_{x y} & n_{x z} \\ \bigcap_{y x} & n_{y y} & n_{y z} \\ \bigcap_{z x} & n_{z y} & n_{z z}\end{array}\right]$

the $x$-component of the vector Div $\cap$ is given by eqn. (9).

$(\operatorname{Div} \cap)_{x}=(\partial / \partial x) \cap_{x x}+(\partial / \partial y) \cap_{y x}+(\partial / \partial z) \cap_{z x}$

Similar equations can be written for the $y$ - and $z$-directions, but they are not of interest here. Eqn. (7) will now be modified to include changes caused by the electric current.

\section{Momentum conservation in the presence of electric current}

We now postulate that the presence of electric current will cause additions to the shear stresses, $\cap_{i j}$; there will be an excess stress tensor. This statement is similar to the postulate of a mass flux in excess of diffusion when electric current is passing electrolyte solutions. ${ }^{1,2,11}$ The electric current density is the vector $\boldsymbol{j}=\left\{j_{x}, j_{y}, j_{z}\right\}$, and the excess contributions in each direction are expected to be proportional to the components of $j$. The excess contribution defines the transference factor $\tau_{x i, x j}$ for transfer of momentum from the $i$ direction to the $j$ direction due to electric current. We write the matrix of the excess components as eqn. (10), with $\tau_{x x}=\tau_{y y}=\tau_{z z}$; cf. eqn. (5). We

$\bar{\pi}=\left[\begin{array}{lll}\tau_{x x} j_{x} & \tau_{x y}\left(j_{x}+j_{y}\right) / 2 & \tau_{x z}\left(j_{x}+j_{z}\right) / 2 \\ \tau_{y x}\left(j_{y}+j_{x}\right) / 2 & \tau_{y y} j_{y} & \tau_{y z}\left(j_{y}+j_{z}\right) / 2 \\ \tau_{z x}\left(j_{z}+j_{x}\right) / 2 & \tau_{z y}\left(j_{z}+j_{y}\right) / 2 & \tau_{z z} j_{z}\end{array}\right]$

assume that the excess stress tensor is symmetric. The tensor $\bar{\pi}$ assigns a force $d \boldsymbol{F}$ to the vector $\mathrm{d} \boldsymbol{A}$ in the same way as given in eqns. (3a-3c) (Fig. 2). The tensor $\bar{\pi}$ is constructed from the vector $j$ according to eqn. (10). The three columns formed as on the right-hand side of eqns. (3) are the momentum fluxes through the areas $\mathrm{d} A_{x}, \mathrm{~d} A_{y}$ and $\mathrm{d} A_{z}$ (see below). We may also write the excess stress tensor in a form similar to eqn. (4), eqn. (11), where the diagonal

$\bar{\pi}=\sigma^{\prime}\{\mathbf{1}\}+\bar{\pi}^{\prime}$

elements make up the quantity $\sigma^{\prime}$, given by eqn. (12), and

$\sigma^{\prime} \equiv \sigma\left(j_{x}+j_{y}+j_{z}\right)=\tau_{x x}\left(j_{x}+j_{y}+j_{z}\right) / 3$

the matrix $\bar{\pi}$ contains the elements shown in eqn. (13).

$\bar{\pi}^{\prime}=\left[\begin{array}{lll}\tau_{x x} j_{x}-\sigma^{\prime} & \tau_{x y}\left(j_{x}+j_{y}\right) / 2 & \tau_{x z}\left(j_{x}+j_{z}\right) / 2 \\ \tau_{y x}\left(j_{y}+j_{x}\right) / 2 & \tau_{y y} j_{y}-\sigma^{\prime} & \tau_{y z}\left(j_{y}+j_{z}\right) / 2 \\ \tau_{z x}\left(j_{z}+j_{x}\right) / 2 & \tau_{z y}\left(j_{z}+j_{y}\right) / 2 & \tau_{z z} j_{z}-\sigma^{\prime}\end{array}\right]$

$\sigma^{\prime}$ and $\sigma$ refer to the mean value of the three components of $j$. The different components of the excess stress tensor, $\bar{\pi}$, can be understood as follows. The sum of the diagonal elements, $\sigma^{\prime}$, in eqn. (12) may be regarded to cause a macroscopic motion of the liquid, and thus a change in velocity of $\mathrm{d} V$, due to electric current. They can add an extra contribution over and above the effect of the hydrostatic pressure. The off-diagonal elements can add extra contributions to the shear stresses, which, however, are not diffusive. This also may cause motion of the liquid.

The electric current is chosen to have an $x$-component only, $\boldsymbol{j}=\left\{j_{x}, 0,0\right\}=\{j, 0,0\}$. Thus from eqn. (12) we have eqn. (14). We also have eqn. (15). The trace of the matrix 
$\sigma^{\prime}=\tau_{x x} j_{x} / 3=\sigma_{j}$

$\bar{\pi}^{\prime}=\left[\begin{array}{lll}2 \tau_{x x} j / 3 & \tau_{x y} j / 2 & \tau_{x z} j / 2 \\ \tau_{y x} j / 2 & -\tau_{x x} j / 3 & 0 \\ \tau_{z x} j / 2 & 0 & \tau_{x x} j / 3\end{array}\right]$

is zero, as it should be by definition. In order to find the equation of motion we need the tensor divergence of $\bar{\pi}$.

\section{Divergence values of the excess stress tensor}

Note first that $I=\int j \mathrm{~d} A$ is constant, where $A$ is the electric current and $A$ is the cross-section of the system. Thus we have $\operatorname{div} \boldsymbol{j}=0$. From eqn. (15) the divergence components of $\bar{\pi}^{\prime}$ are given by eqn. (16). By combining eqn. (16) with

$$
\left(\operatorname{Div} \bar{\pi}^{\prime}\right)_{x}=(\partial / \partial x)\left(2 \tau_{x, j} j / 3\right)+(\partial / \partial y)\left(\tau_{y x} j / 2\right)+
$$

$$
(\partial / \partial z)\left(\tau_{z . j} / 2\right)
$$

$\left(\operatorname{Div} \bar{\pi}^{\prime}\right)_{v}=(\partial / \partial x)\left(\tau_{v j} j / 2\right)-(\partial / \partial y)\left(\tau_{x, j} j / 3\right)$

$\left(\operatorname{Div} \bar{\pi}^{\prime}\right)_{z}=(\partial / \partial x)\left(\tau_{x j} j / 2\right)-(\partial / \partial z)\left(\tau_{x j} j / 3\right)$

$\sigma^{\prime}\{\mathbf{1}\}$ according to eqn. (12), we obtain eqn. (17). As

$$
(\operatorname{Div} \bar{\pi})_{x}=3(\partial / \partial x)(\sigma j)+(\partial / \partial y)\left(\tau_{y, j}\right) / 2+
$$

$$
(\partial / \partial z)\left(\tau_{z: j}\right) / 2
$$

$(\operatorname{Div} \bar{\pi})_{y}=(\partial / \partial x)\left(\tau_{x y} j\right) / 2$

$(\operatorname{Div} \bar{\pi})_{z}=(\partial / \partial x)\left(\tau_{x j} j\right) / 2$

already indicated above, the total excess momentum per unit time and area is the excess force per unit area. Thus the force can be written in analogy with eqn. (3), by using eqns. (11), (14) and (15), as eqn. (18). The excess force in

$$
\begin{aligned}
\mathrm{d} F_{x} & =\left(\tau_{x j} j\right) \mathrm{d} A_{x}+\left(\tau_{x y} j / 2\right) \mathrm{d} A_{y}+\left(\tau_{x j} j / 2\right) \mathrm{d} A_{z} \\
\mathrm{~d} F_{y} & =\left(\tau_{y, j} j / 2\right) \mathrm{d} A_{x} \\
\mathrm{~d} F_{z} & =\left(\tau_{z . j} j / 2\right) \mathrm{d} A_{x}
\end{aligned}
$$

the $x$-direction is the vector given by eqn. (19a), which is equivalent to the excess momentum passing per unit time and area in the $x$-direction. Similarly, for the $y$ - and $z$-directions we have eqns. (19b) and (19c). These vectors

$$
\boldsymbol{F}(x)=j\left\{\tau_{x x}, \tau_{x y} / 2, \tau_{x z} / 2\right\}
$$

$\boldsymbol{F}(y)=j\left\{\tau_{x y} / 2,0,0\right\}$

$$
\boldsymbol{F}(z)=j\left\{\tau_{x z} / 2,0,0\right\}
$$

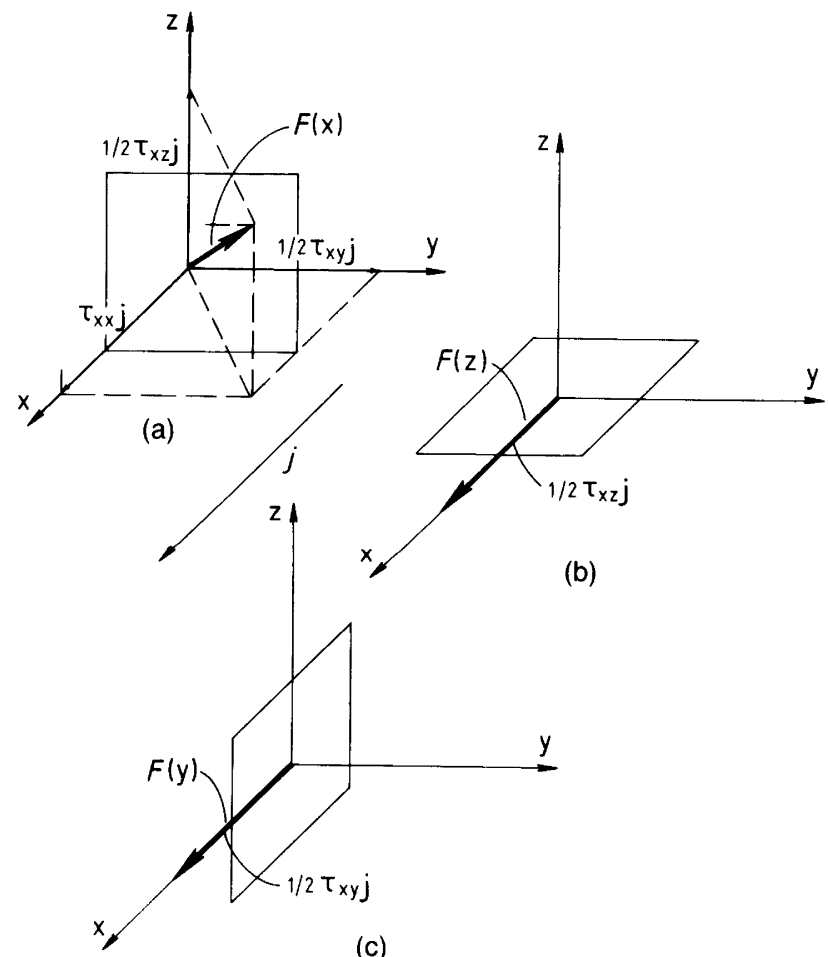

(c)

Fig. 3. Excess momentum flux components for the $x$-, $y$ - and $z$-directions in the presence of electric current density $\boldsymbol{j}=\{j, 0,0\}$ : (a) momentum passing per unit time through an area $\mathrm{d} A_{\mathrm{x}} ;(\mathrm{b})$ momentum passing per unit time through an area $\mathrm{d} A_{z}$; (c) momentum passing per unit time through an area $\mathrm{d} A_{y}$. Note that we have $\tau_{z x}=\tau_{x z}$ and $\tau_{y x}=\tau_{x y}$.

are illustrated in Figs. 3a-3c. The components of Div $\pi$ are the divergences of the vectors $F(x), F(y)$ and $F(z)$ according to eqn. (19).

Momentum, $\varrho v$, can only be supplied or received at interfaces, not in a homogeneous bulk phase. The derivatives $\partial \tau_{x i, x j} / \partial x_{i}$ are non-zero at places where the material changes. In the chosen geometry, momentum can be taken up at the electrodes, which primarily are $z y$-planes, and at the walls of the $x y$-planes. The electrodes are not infinitely thin, thus momentum can be transferred between their surface and the liquid and partly via the electrode edges, which are facing the $y$ - and $z$-direction. The situation is illustrated in Fig. 4.

We shall first discuss more closely the situation at the electrodes. If positive momentum is taken away from one electrode and delivered to the liquid, there must be a deficit in the term $\varrho d v / \mathrm{d} t$ belonging to this electrode. This is illustrated by the broken arrow in Fig. 4 for the left-hand electrode. A deficit means a loss, i.e. movement has a negative direction. The momentum loss is quenched by the electrode; it is converted into mechanical tension, oscillations are damped out, and it remains potential energy. At the right-hand electrode, momentum may be given from the liquid to the electrode, as indicated by the broken arrow pointing to the right, but some part of the momentum gained by the liquid at the left-hand electrode may 


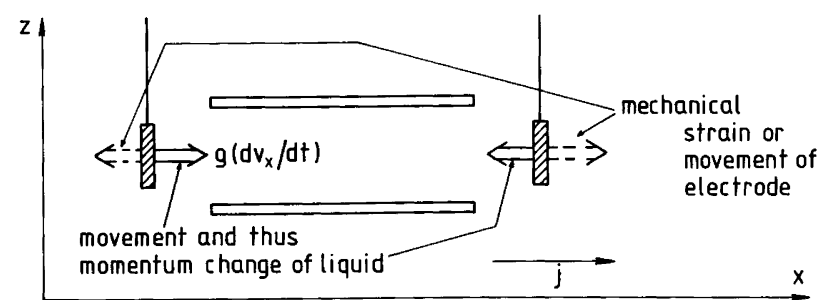

Fig. 4. Momentum transfer at the electrodes. A xz-cross-section is given: Contributions to $\varrho \mathrm{d} v_{x} / \mathrm{d} t$ are indicated. Contributions to $\varrho d v_{y} / \mathrm{d} t$ and $\varrho d v_{z} / \mathrm{d} t$ at the electrode edges are also possible (see text).

also be kept by the solution. This means that the solution will move. Altogether this is a consequence of the fact that the $x$-components of the force $(\partial / \partial x)\left(\tau_{x x} j\right) / 3=\partial(\sigma j) \partial x$ are non-zero at the two electrode surfaces parallel to the $z y$-plane. Unbalanced $y$ - and $z$-components of the force, $(\partial / \partial x)\left(\tau_{x y} j\right)$ and $(\partial / \partial x)\left(\tau_{x z} j\right)$ [eqns. $(17 \mathrm{~b})$ and $\left.(17 \mathrm{c})\right]$, act at the electrode edges only, because $y$ - and $z$-directions are not defined at the surface $z y$-plane.

The values of the normal force $\partial(\sigma j) / \partial x$, as well as the components $(\partial / \partial y)\left(\tau_{y x} j\right) / 2$ and $(\partial / \partial z)\left(\tau_{z x} j\right) / 2$, are, however, zero in the homogeneous bulk; the uniform bulk cannot receive or supply momentum. At the planes the term $(\partial / \partial z)\left(\tau_{z . x} j\right) / 2$ exists; consequently momentum may be transferred from the walls into the liquid in the $x$-direction.

If the momentum given off at the right-hand side is larger than that taken up by the left-hand side, then the direction of motion is from right to left. In a vessel which is bound at both ends, $x=0, x=1$, the momentum of the solution is immediately balanced by the hydrostatic pressure. No sufficient tangential stress can be maintained in the liquid in this geometry, so the liquid flows back. Without such boundaries the liquid movement would be damped by shear stresses.

\section{Equation of motion for viscous flow in the presence of electric current}

The effect of electric current can now be included in the equation of motion [eqn. (7)]. We shall confine ourselves to the $x$-component of the divergence of $\pi$, since this gives the main effects. We thus neglect effects at electrode edges. For the boundaries (electrodes and planes) we have eqn. (20a) for the local rate of change of $\mathrm{Q}\left(\mathrm{d} v_{x} / \mathrm{d} t\right)=-\mathrm{d} p / \mathrm{d} x+\eta\left(\partial^{2} v_{x} / \partial z^{2}\right)-3 \partial(\sigma j) / \partial x-$

$$
(\partial / \partial z)\left(\tau_{z x} j\right) / 2
$$

momentum. With the approximation of $j=$ constant, this gives eqn. (20b). As the last two terms are zero in the bulk,

$$
\begin{gathered}
\mathrm{\varrho}\left(\mathrm{d} v_{x} / \mathrm{d} t\right)=-\mathrm{d} p / \mathrm{d} x+\eta\left(\partial^{2} v_{x} / \partial z^{2}\right)-3(\partial \sigma / \partial x) j- \\
\left(\partial \tau_{z x} / \partial z\right) j / 2
\end{gathered}
$$

eqn. (7) can be used unaltered for the bulk. In order to integrate eqn. (20), we need the transference factors, $\sigma$ and $\tau_{z x}$, as functions of the $z$-coordinate. Lacking other knowledge we shall assume the functional dependence for $\tau_{z x}$ given by eqn. (21), where $\delta z$ represents a small layer at the

$\tau_{z x}(z)=\tau_{z x}-\tau_{\mathrm{zx}}\left(z-z_{0}+\delta z\right) / \delta z$

boundary. The function is illustrated in Fig. 5. It follows that eqn. (22) holds. Likewise at the electrodes, we have eqn. (23) (Fig. 5). The subscripts a and c indicate anode and cathode materials, respectively. The superscripts indicate solution values around the anode or cathode.

$\mathrm{d} \tau_{z x} / \mathrm{d} z=-\tau_{\mathrm{zx}} / \delta z$

$(\mathrm{d} \sigma / \mathrm{d} x)_{\mathrm{a}}=\left(\sigma^{\mathrm{a}}-\sigma_{\mathrm{a}}\right) / \delta x=-\Delta \sigma_{\mathrm{a}} / \delta x$

$(\mathrm{d} \sigma / \mathrm{d} x)_{\mathrm{c}}=\left(\sigma_{\mathrm{c}}-\sigma^{\mathrm{c}}\right) / \delta x=\Delta \sigma_{\mathrm{c}} / \delta x$

\section{The electric work in the absence of pressure gradients}

The purpose of integrating the equations of motion is to find the mechanical work done by the system per unit time, which is equivalent to the electrical work. Consider first the contributions from the transference factors in eqn. (20b)

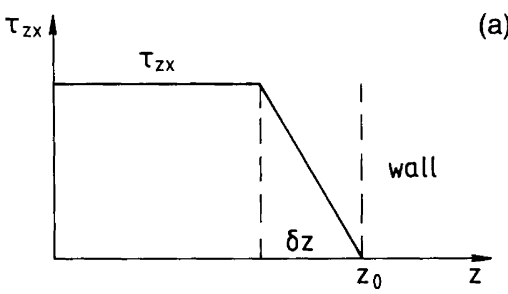

(a)
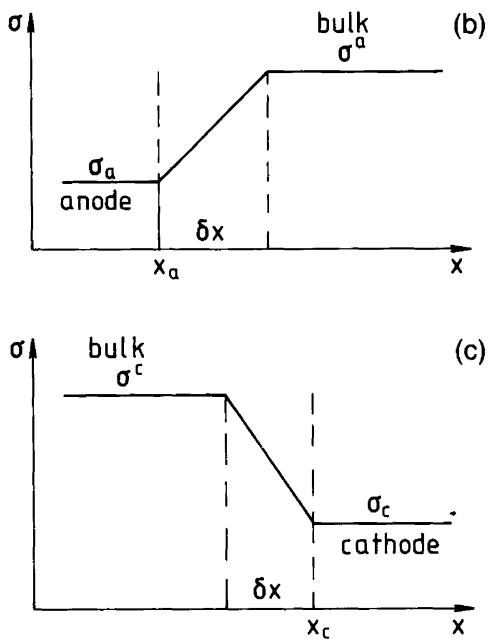

Fig. 5. Momentum transference factors as functions of coordinates: (a) the transference factors $\tau_{z x}$; (b) and (c) the transference factor $3 \sigma$ at the anode and at the cathode. 
when $\mathrm{d} p / \mathrm{d} x=0$. Within the small range $z_{0}-\delta z<z<z_{0}$, at the upper plane we have $(\mathrm{d} \sigma / \mathrm{d} x)=0$. Assume that the breadth of the plane in the $y$-direction is $a$. We multiply eqn. (20b) with $a$ and integrate to get eqn. (24). A similar

$$
\int_{z_{1}-\partial z}^{z_{11}} a \varrho\left(\mathrm{d} v_{x} / \mathrm{d} t\right) \mathrm{d} z=\int_{z_{1}-\partial z}^{z_{10}} a \eta\left(\partial^{2} v_{x} / \partial z^{2}\right) \mathrm{d} z+a \tau_{z x} j / 2
$$

equation holds for the lower plane at $z=-z_{0}$. The effects of the regions close to the two planes are included in eqn. (25). By extending the integration from $-z_{0}$ to $z_{0}$ we

$a \int_{-z_{0}}^{z_{0}} \varrho\left(\mathrm{d} v_{x} / \mathrm{d} t\right) \mathrm{d} z=a \int_{-z_{0}}^{z_{0}} \eta\left(\partial^{2} v_{x} / \partial z^{2}\right) \mathrm{d} z+a \tau_{z x} j$

have obtained an equation of motion for a plane of liquid perpendicular to the transport direction, with height $2 z_{0}$, breadth $a$ and thickness $\mathrm{d} x$. In the limit of small velocities, the second-order term containing the viscosity is negligible. Eqn. (25) then reduces to eqn. (26), where $\bar{u}_{x}$ is the

$2 a z_{11} \mathrm{Q}(\mathrm{d} \bar{u}, / \mathrm{d} t)=a \tau_{: j} j$

average of $v_{x}$ over the cross-section. The left-hand side of eqn. (26) is a force per unit length. The mechanical work to be given to the system per unit time by moving the column of liquid can be obtained by multiplying eqn. (26) by $\bar{u}_{x} l$ as in eqn. (27a), where $l$ is the length of the system where

$$
\mathrm{d} W_{\text {mech }} / \mathrm{d} t=\bar{u}_{x} 2 a z_{0} l \varrho\left(\mathrm{d} \bar{u}_{x} / \mathrm{d} t\right)=\tilde{u}_{x} l a \tau_{z x} j
$$

electric current is flowing. In addition to the work done at the walls, we have work done at the electrodes. By integrating eqn. (20b) first with respect to $z$ and then with respect to $x$ using eqn. (23), we get eqn. (27a) in the form of eqn. (27b), which is the total work per unit time supplied

$$
\begin{aligned}
& \mathrm{d} W_{\text {mech }} / \mathrm{d} t= \bar{u}_{x} 2 a z_{0} l \varrho\left(\mathrm{d} \bar{u}_{x} / \mathrm{d} t\right)= \\
& \bar{u}_{x} a\left[-6 z_{0}\left(\Delta \sigma_{\mathrm{c}}-\Delta \sigma_{\mathrm{a}}\right) j+l \tau_{z x} j\right]
\end{aligned}
$$

to the system. In the present system, work is supplied as electrical work, EI. This gives eqn. (28), where $I$ is the

$\mathrm{d} W_{\text {mech }} / \mathrm{d} t=\mathrm{d} W_{\mathrm{e}} / \mathrm{d} t=-E 2 a z_{0} j=-E I$

electric current. In the absence of dissipative effects $(I \approx 0)$, $E$ is the EMF of the system. By comparing eqns. (27b) and (28), we get eqn. (29).

$$
E=\left[-\tau_{z x} l / 2 z_{0}+3\left(\Delta \sigma_{\mathrm{c}}-\Delta \sigma_{\mathrm{a}}\right)\right] \bar{u}_{x}
$$

The volume flux of the liquid is equal to $J_{\mathrm{v}}=2 z_{0} a \bar{u}_{x}=$ $A \bar{u}_{x}$, where $A$ is now the cross-section of the moving phase. By introducing $J_{\mathrm{v}}$ we get our result in a form which can be related to experiments: eqn. (30). This equation has not
$E=\left(J_{\mathrm{v}} / A\right)\left[-\tau_{z x} / / 2 z_{0}+3\left(\Delta \sigma_{\mathrm{c}}-\Delta \sigma_{\mathrm{a}}\right)\right]$

yet been derived. Essentially, it describes friction electricity. In addition to pressure gradients (and chemical potential gradients), viscous frictional and gravitational work are not relevant for the derivation of eqn. (30), which describes the EMF of a system where different kinds of matter have a velocity relative to one another. Usually a nonstationary state is assumed. Assume that we start with the system at rest. An electric current is then applied. During the initial phase there is a variation in liquid movement, $\dot{u}_{x}$, caused by the divergence of the components of the excess stress tensor. Electrical work has been applied to the system and is transformed into mechanical work (kinetic energy). Alternatively, the variation in $\bar{u}_{x}$ back to rest is able to generate an EMF according to eqn. (30). The relative size of the effects, viscous and reversible excess friction, will determine the possibility of converting mechanical energy into electric or thermal energy.

\section{Friction electricity, a further discussion}

Consider again the system in Fig. 1. Neglect for the moment effects caused by the stress tensor. The liquid flows with velocity $\bar{u}_{x}$. There are no pressure gradients. The changes in linear momentum are expressed by eqn. (27b) for these conditions [eqn. (31)], where $L$ is the total length

$\left(2 a z_{0} L\right) \varrho\left(\mathrm{d} \bar{u}_{x} / \mathrm{d} t\right)=-6 a z_{0}\left(\Delta \sigma_{\mathrm{c}}-\Delta \sigma_{\mathrm{a}}\right) j+a l \tau_{z x} j$

of the column of liquid, including parts beyond electrodes, and $\mathrm{d} \bar{u}_{x} / \mathrm{d} t$ refers to a mean value along the $z$-axis. Eqn. (31) tells us that the change in linear momentum is determined by the geometry and types of materials and by the electric current density.

Momentum conservation states that momentum is taken up by the rigid surroundings when the liquid looses speed at the electrodes or at the walls. The momentum lost by the liquid is supplied to the excess momentum flux tensor, $\bar{\pi}$. This produces the electric current density $j$. Here in particular it is the $z$-component of the vector which represents the flux of the scalar quantity the $x$-component of the momentum. This excess flux itself may have any value; its absolute value is not physically defined, only the decrease (or increase) at the boundaries, $\tau_{z x} j$, is well defined (Fig. 5). At the electrodes there are also divergences of the excess momentum flux. The flowing liquid with its loss of linear momentum produces a contribution to the electric current density. If $\left(\Delta \sigma_{c}-\Delta \sigma_{a}\right)=0$, the consumption and delivery of momentum at the electrodes are exactly balanced. When the type and state of the two electrodes and the processes in the adjacent solutions are the same, this condition will be fulfilled.

The occurrence of $j$ on the right-hand side of eqn. (31) means that momentum consumption does not happen at random; it is strictly correlated with other processes. 
Therefore $\boldsymbol{j}$ is the coherence parameter,${ }^{1}$ here for momentum changes. Since momentum conservation requires that $\mathrm{d}(m v) / \mathrm{d} t=-\mathrm{d}\left(m^{\prime} v^{\prime}\right) / \mathrm{d} t$ when two masses $m$ and $m^{\prime}$ interact, a large value for $m^{\prime}$ means that $v^{\prime}$ is small. When $m^{\prime}$ stands for the equipment (electrodes and walls), then $v^{\prime}$ will be almost zero. There is only a slight vibration about the new equilibrium position of the equipment.

\section{Electric work from friction electricity}

The rate of energy loss which corresponds to the loss of linear momentum is derived from eqn. (29) and given by eqn. (32). Consider next the electric work which can be

$E I=I\left[-\tau_{z x} l / 2 z_{0}+3\left(\Delta \sigma_{\mathrm{c}}-\Delta \sigma_{\mathrm{a}}\right)\right] \bar{u}_{x}$

delivered by the system per unit time, $-E I$. The electrodes can be connected in principally two different ways, in an open or closed torus. ${ }^{1}$ To start we say that the electric work is delivered to a "black box". As the first possibility, let the black box be a conductor with resistance $R$. Then all the energy per unit time delivered by the flowing liquid occurs as Joule heat in the conductor. If the "black box" is a capacitor, the electric work per unit is given by eqn. (33),

$-E I=R I^{2}+(I / C) \int I \mathrm{~d} t$

where $C$ is the capacitance of the capacitor. If the capacitor is fully charged, $I=0$. No energy can then be obtained from the moving liquid. The liquid flows without retardation. For comparison, consider the expression for the galvanic cell given by eqn. (27) in Ref. 1 [eqn. (34)].

$-E I=I\left(\mu_{\mathrm{AB}}-\mu_{\mathrm{A}}-\mu_{\mathrm{B}}\right) / F=R I^{2}+(I / C) \int I \mathrm{~d} t$

This expression is of the same structure as eqn. (33) with eqn. (32). The difference is the origin of $E$. In eqn. (34) the electric current is given by $\mathrm{d} n_{\mathrm{AB}} / \mathrm{d} t$, i.e. by the mass production of $\mathrm{AB}$. The composition of the system changes and gives rise to a change in $E I$. The value of $E$ is not strictly constant: it depends on the amount of matter which has reacted. In the electrokinetic experiment $I$ is initially given by $\mathrm{d}\left(m v_{x}\right) / \mathrm{d} t$, thus if $I$ is non-zero, the momentum of the liquid becomes smaller, i.e. $\bar{u}_{x}$ becomes smaller; finally $\bar{u}_{x}=0$, and consequently the EMF has ceased to exist. There is no longer electrical energy: all mechanical and electrical energy has been converted into thermal energy. This is the same as for a galvanic cell: the cell yields energy, i.e. $E \neq 0$, but the composition of the system approaches that of chemical equilibrium, and eventually $E=0$. So, in eqns. (32) and (33) we must not choose too large a value of $C$. The momentum of the liquid must not be used up before the capacitor has attained a voltage which makes $I=0$. With $I=0$ the electric energy, which can be extracted from the system, is stored in the capacitor. The liquid, however, continues to move with a velocity given by eqn. (35).
$\bar{u}_{x}=E /\left[-\tau_{z x} l / 2 z_{0}+3\left(\Delta \sigma_{\mathrm{c}}-\Delta \sigma_{\mathrm{a}}\right)\right]$

We have so far discussed a torus construction with electrodes acting, among other things, as boundaries for the flowing liquid. The length of the liquid between the electrodes connected to the black box is $l$, but the total length of the flowing liquid is $L$. The end pieces of the container can be regarded as effective electrodes connected to a capacitor, but the capacitance of this is almost zero. The torus is closed through the vector $\mathrm{d} D / \mathrm{d} t$, the displacement current. No electric current is flowing immediately after the onset of the movement. However, electric energy persists as long as the liquid moves. This is the production of friction electricity in the more conventional sense. The liquid may of course be replaced by an insulator or a solid bar.

\section{The streaming potential}

The presence of a pressure gradient may lead to stationarystate conditions. Assume that such conditions are obtained, so that the left-hand side of eqn. (20b) becomes zero. We are left to perform the integration over the spatial region of the liquid. First we integrate over the boundary regions. The result is given by eqn. (36) [cf. eqns. (21)-(25)]. In the

$$
\begin{gathered}
0=-a \int_{\delta x}[\mathrm{~d} p / \mathrm{d} x] \mathrm{d} x+2 l a \int_{z_{0}-\delta z}^{z_{0}} \eta\left(\partial^{2} \bar{v}_{\mathrm{x}} / \partial z^{2}\right) \mathrm{d} z- \\
6\left(\Delta \sigma_{\mathrm{c}}-\Delta \sigma_{\mathrm{a}}\right) a z_{0} j+a l \tau_{z x} j
\end{gathered}
$$

bulk we have eqn. (37). We assume that the pressure gra-

$0=-2 a z_{0} \int_{0}^{l}[\mathrm{~d} p / \mathrm{d} x] \mathrm{d} x+a l \int_{-z_{0}}^{z_{0}} \eta\left(\partial^{2} \overline{\mathrm{v}}_{\mathrm{x}} / \partial z^{2}\right) \mathrm{d} z$

dient $\mathrm{d} p / \mathrm{d} x$ is constant. The value of the first integral is $\Delta p$ $=|p(x=l)-p(x=0)|$. The constant value of the first term can be used to define the hydrodynamic resistance $R_{\mathrm{v}}$ and the mean velocity through eqn. (38).

$J_{\mathrm{v}}=A \bar{u}_{x}=\Delta p / R_{\mathrm{v}}$

The first two terms of eqn. (36) are zero because the ranges of integration are vanishingly small. The terms containing $j$ in eqn. (36) vanish as well, because $j=0$ in a stationary open circuit. Yet the factor of $2 a z_{0} j$ in eqn. (36) is the quantity $-E / \bar{u}_{x}$ [eqn. (29)]. We shall derive the well known formula for the EMF in systems with pressure gradients, the streaming potential. The streaming potential is defined by $(E / \Delta p)_{l \approx 0}$, and is obtained by introducing eqn. (38) into eqn. (29). This gives eqn. (39). The streaming

$(E / \Delta p)_{l \approx 0}=\left[-\tau_{z x} / / 2 z_{0}+3\left(\Delta \sigma_{\mathrm{c}}-\Delta \sigma_{\mathrm{a}}\right)\right] / A R_{\mathrm{v}}$

potential represents the electric work to be gained from the 
system by the effect of a given pressure gradient in a stationary state.

\section{Onsager reciprocal relations are not necessary for the derivation of certain relations}

It has long been known that certain relations exist among observables in the systems with which we are dealing. The Saxén relations equate the streaming potential and the electro-osmotic flux, and the streaming current and electroosmotic pressure. These relations are usually derived by applications of Onsager reciprocal relations in irreversible thermodynamics..$^{7-9}$ We shall see that the relations follow from the basic eqn. (30).

The expression for the streaming potential has already been derived [eqn. (39)]. The electro-osmotic flux will be derived below. We start from the equation of motion, eqn. (20b). Again the assumption of a stationary state is introduced. This makes the left-hand side of the equation equal to zero. In this type of experiment there is no pressure gradient. We integrate first with respect to $x$ and next we perform the integration between $z_{0}$ and $-z_{0}$. The result is eqn. (40). For stationary state conditions in the bulk,

$0=-6 z_{0}\left(\Delta \sigma_{\mathrm{c}}-\Delta \sigma_{\mathrm{a}}\right) j+\mid l \underset{-z_{0}}{\sum_{0}} \partial^{2} v_{x} / \partial z^{2}+l \tau_{z x} j$

eqn. (7) can be integrated to give eqn. (41). This relation

$\left.2 a z_{0} \Delta p=\prod_{-z_{0}}^{z_{0}} a l \eta \partial^{2} v_{x} / \partial z^{2}\right)$

holds true in the absence of any electric effect, but we also use it for the definition of $R_{\mathrm{v}}$ in the absence of pressure gradients. According to eqns. (37) and (38) we then have eqn. (42). The volume flux $J_{v}$ from eqn. (41) can be

$\left.2 z_{0} R_{v} J_{v}=\prod_{-z_{0}}^{z_{0}} l \eta \partial^{2} v_{x} / \partial z^{2}\right)$

introduced into eqn. (40) to give eqn. (43). By replacing $j$

$2 z_{0} R_{\mathrm{v}} J_{\mathrm{v}}=6 z_{0}\left(\Delta \sigma_{\mathrm{c}}-\Delta \sigma_{\mathrm{a}}\right) j-\tau_{z x} l j$

in eqn. (43) by $I / A$, an expression for the electro-osmotic flux is obtained, identical to the expression for the streaming potential, eqn. (39), which proves the Saxèn relations.

The streaming current is defined for short-circuit conditions when $E=R I$ (no reversible electric work is performed by the system). The short-circuit current produces Joule heat, $R I^{2}$, where $R$ is the electric resistance of the system. The Joule heat was neglected in the derivation of eqns. (29) and (30). When the electric work is zero, the term $R I$ is left on the left-hand side of eqn. (30). It corresponds to a situation in which the term containing the integral $\int I \mathrm{~d} t$ is zero $[C \rightarrow \infty$ in eqn. (34)]. By introducing this into eqn. (29), we obtain eqn. (44), which gives eqn. (45).

$$
\begin{aligned}
& I=\left(J_{\mathrm{v}} / R A\right)\left[3\left(\Delta \sigma_{\mathrm{c}}-\Delta \sigma_{\mathrm{a}}\right)-\tau_{z x} l / 2 z_{0}\right] \\
& \left(I / J_{\mathrm{v}}\right)_{E=0}=\left[3\left(\Delta \sigma_{\mathrm{c}}+\Delta \sigma_{\mathrm{a}}\right)-\tau_{z x} l / 2 z_{0}\right] / R A
\end{aligned}
$$

The electro-osmotic pressure is measured for an electric current which produces a stationary-state pressure that makes $J_{v}=0$. By integrating eqn. (7), together with eqn. (20b), for these conditions we obtain eqn. (46). Since

$0=-2 z_{0} \Delta p+\left[6 z_{0}\left(\Delta \sigma_{\mathrm{c}}-\Delta \sigma_{\mathrm{a}}\right)-\tau_{z x} l\right] I / A$

$J_{v}=0$, the viscosity term vanishes. By introducing the relation $E \approx R I$ into eqn. (46), we obtain as a result for $(\Delta p / E)_{J_{\mathrm{v}}=0}$ the right-hand side of eqn. (45), which also proves the Saxèn relations.

We have thus been able to derive the Saxèn relations without invoking the Onsager reciprocal relations. The conclusion that may be drawn from this result is that the validity of the Onsager reciprocal relations is consistent with the electric work method. Their proof is given indirectly through this, since they must be fulfilled in order to make the theory of irreversible thermodynamics obey the conservation laws.

\section{The electric work method compared to conventional theories of electrokinetic effects}

The book by Koryta and Dvorak may be taken as a typical text dealing with electrokinetic phenomena. ${ }^{12}$ The electric field, $\mathrm{d} \varphi / \mathrm{d} x$, in the direction of the axis of the pores is expressed by the internal friction of the liquid in these texts as eqn. (47). This equation describes a fundamentally dif-

$\mathrm{d} \varphi / \mathrm{d} x=\eta\left(\partial^{2} v_{x} / \partial z^{2}\right)$

ferent interpretation of electrokinetic phenomena than the one given here. The right-hand side of eqn. (47) expresses an irreversible phenomenon, uncoordinated with the electric work performed. We, on the other hand, assign the electric work to a reversible property of the system and the coherence of several phenomena.

This difference in interpretation has its analogy in the interpretation of liquid junction potentials. Conventionally this potential is explained as "a diffusion potential" caused by diffusing ions. The electric work method relates the liquid junction potential to reversible mass and energy changes of neutral compounds.

The diffusing ions cause a charge separation, giving rise to an electrostatic potential in the conventional theory. In electrokinetic systems the electrostatic potential is caused by the zeta potential. This potential drop has its direction perpendicular to the direction of the electric current. It is the variation of the zeta potential in the direction of the 
electric current which gives rise to a charge separation and thus an observable electric potential difference. This model, even if self-consistent, relies on the construction of the zeta potential, an unmeasurable quantity. Thus the conventional theory cannot be checked by experiments. We consider this a major drawback of this theory.

According to the theory of irreversible thermodynamics, the streaming potential is due to the transference numbers of water and salt in the membrane, ${ }^{9} t_{\mathrm{H}_{2} \mathrm{O}}$ and $t_{\mathrm{NaCl}}$. In our particular system we have eqn. (48), where $\Delta V_{\mathrm{el}}$ represents

$(E / \Delta p)_{l \approx 0}=-\left(t_{\mathrm{NaCl}} V_{\mathrm{NaCl}}+t_{\mathrm{H}_{2} \mathrm{O}} V_{\mathrm{H}_{2} \mathrm{O}}\right)-\Delta V_{\mathrm{el}}$

volume changes at the electrodes per Faraday. This equation expresses the EMF as the result of mass movement due to electric current. Usually the transfer of water is related to the ion transport. Monovalent ions have been taken to carry a smaller amount of water through ion-exchange membranes than divalent ions, which possess a larger field strength. Values for $t_{\mathrm{H}_{2} \mathrm{O}}$ larger than 50 are, however, observed in very leaky biological membranes. ${ }^{13}$ This makes the explanation of water transfer by ion transfer alone unlikely. We see that the present analysis offers a direct way of explaining data in such cases, through the momentum transference factors. Both eqn. (48) and the expression for the streaming potential given by eqn. (39) express the EMF by essentially two effects, the electrode surface effects and the effects of the planar walls. Our analysis emphasizes that the effects of salt and water on the momentum transference factor of the wall are experimentally inseparable.

\section{Conclusions}

We have seen above that all the major formulas of electrokinetics may been derived from a new basis, that given by the equation of conservation of momentum in a hydrodynamic system which conducts electric current. In addi- tion, the phenomenon of friction electricity has been studied in a new light. The experimental parameters used to describe friction electricity are the momentum transference factors $\tau_{x i, x j}$. The determination of these parameters will be a future goal of our studies.

Acknowledgements. Support from The Royal Norwegian Foundation for Sciences and Humanities to G.H.H. is acknowledged.

\section{References}

1. Hertz, H. G. and Ratkje, S. K. Acta Chem. Scand. 44 (1990) 542.

2. Hertz, H. G. and Ratkje, S. K. Acta Chem. Scand. 44 (1990) 554.

3. Hertz, H. G. and Ratkje, S. K. J. Electrochem. Soc. 136 (1989) 1692.

4. Hertz, H. G. and Ratkje, S. K. J. Electrochem. Soc. 136 (1989) 1698.

5. Bockris, J. O'M. and Reddy, A. K. N. Modern Electrochemistry, Plenum Press, New York 1970.

6. de Groot, S. R., Mazur, P. and Overbeek, J. J. Chem. Phys. 20 (1952) 12.

7. Fitts, D. D. Nonequilibrium Thermodynamics, McGraw-Hill, New York 1962.

8. Haase, R. Thermodynamics of Irreversible Processes, Addison-Wesley, New York 1969.

9. Førland, K. S., Førland, T. and Ratkje, S. K. Irreversible Thermodynamics. Theory and Applications, Wiley, Chichester 1988.

10. Kreuzer, H. Nonequilibrium Thermodynamics and its Statistical Foundations, Clarendon Press, Oxford 1981.

11. Hertz, G. Electrochemistry, Springer, Berlin 1980.

12. Koryta, J. and Dvorak, J. Principles of Electrochemistry, Wiley, Chichester 1987.

13. Lyslo, A., Kvernes, S. and Ratkje, S. K. Exp. Eye Res. 32 (1981) 673.

Received April 30, 1990. 